\title{
Educação em tempos de pandemia: o contexto do ensino médico no Brasil
}

RESUMO:

Sales José Lopes Gonçalves Rosa ${ }^{1}$

Nathalia Bandeira de Almeida ${ }^{2}$

Leandra de Jesus Sonego ${ }^{3}$

Ana Clara Santos Mundim ${ }^{4}$

Júlia Lisboa Mendes ${ }^{5}$

Marcela Lemes Marquez da Cunha ${ }^{6}$

Verônica Clemente Ferreira ${ }^{7}$

O mundo sofreu uma reviravolta em meados de 2020, após o início da pandemia de SARS-CoV-2, em março de 2020. Com a educação médica no Brasil não foi diferente. Os alunosinseridos nas Escolas Médicas brasileiras vêm sofrendo com muitas incertezas. Todas as aulas práticas presenciais foram, inicialmente, proibidas; o retorno destas varia de escola para escola,e a forma como as aulas práticas retornarão constitui uma incógnita para a realidade de alunosdas diferentes faculdades e universidades. Além disso, para as aulas teóricas e aulas práticas nãopresenciais, ainda há o problema da adaptação de alunos e professores às aulas ministradas de modo remoto. Desta forma, o presente estudo avalia, do ponto de vista de discentes e docentes da Medicina, as nuances em torno do complexo tema.

Palavras-chave: Ensino médico. Pandemia. SARS-CoV-2.

\section{Education in times of pandemic: the context of medical education in Brazil}

\begin{abstract}
:
The world suffered a turnaround in mid-2020, after the beginning of the SARS-CoV-2 Pandemic, in March 2020. With medical education in Brazil it was no different. Students enrolledin Brazilian Medical Schools have been suffering from many uncertainties. All face-to-face practical classes were initially prohibited; their return varies from school to school, and the way in which practical classes will return constitutes an unknown factor in the reality of students fromdifferent colleges and universities. In addition, for theoretical and practical classes, there is still the problem of adapting students and teachers to classes taught remotely. Thus, the present studyevaluates, from the point of view of medical students and professors, the nuances surrounding thecomplex theme.
\end{abstract}

Keywords: Medical education. Pandemic. SARS-CoV-2.

\footnotetext{
${ }^{1}$ Acadêmico de Medicina na Universidade Federal de Jataí (UFJ). E-mail: saleslopesgr@discente.ufj.edu.br ${ }^{2}$ Acadêmica de Medicina na Universidade Federal de Jataí (UFJ). E-mail: nathaliaalmeida@discente.ufj.edu.br

3 Acadêmica de Medicina na Universidade Federal de Jataí (UFJ). E-mail: jleandra@ discente.ufj.edu.br

${ }^{4}$ Acadêmica de Medicina na Universidade Federal de Jataí (UFJ). E-mail: anaclarasmundim@ discente.ufj.edu.br

${ }^{5}$ Acadêmica de Medicina na Universidade Federal de Jataí (UFJ). E-mail: julialisboa@ discente.ufj.edu.br

${ }^{6}$ Acadêmica de Medicina na Universidade Federal de Jataí (UFJ). E-mail: marcelalemes@ discente.ufj.edu.br

${ }^{7}$ Docente do curso de Medicina na Universidade Federal de Jataí (UFJ), doutora em Ciências Sociais. E-mail: verosocio@ufj.edu.br
} 


\section{INTRODUÇÃO}

No dia 11 de março de 2020, a Organização Mundial de Saúde (OMS) anunciou o início oficial da pandemia de SARS-CoV-2. O Brasil estabeleceu, como método de contenção da doença, diversas medidas restritivas, destacando-se a paralisação imediata das aulas presenciais em todos os níveis de ensino. Para a continuidade do processo ensinoaprendizagem, o Ministério da Educação (MEC) editou a portaria MEC $\mathrm{n}^{\circ} 1.038$ de 7 de Dezembro de 2020, que "dispõe sobre o retorno às aulas presenciais e sobre caráter excepcional de utilização de recursos educacionais digitais para integralização da carga horária das atividades pedagógicas, enquanto durar a situação de pandemia do novo coronavírus". Entretanto, 365 dias após o início da pandemia, muitas dúvidas pairam sobre o contexto do Ensino Médico remoto de modo emergencial.

Atualmente, há muita discussão das instituições médicas sobre o retorno das aulas práticas presenciais e a sua necessidade vital para o desenvolvimento dos estudantes como futuros profissionais médicos. Paulo Freire (1997, p. 40) afirma que "para compreender a teoria é preciso experienciá-la”. No entanto, num contexto pandêmico, as aulas práticas on-line são a realidade de muitas escolas médicas atualmente, mesmo em discordância com a Associação Brasileira de Educação Médica (ABEM) (2021, p.3) que orienta que "sempre que possível, se retomem as atividades práticas na graduação nas diversas modalidades, incluindo as de resposta à pandemia".

Acerca da complexa problemática, o presente manuscrito analisa o tema a partir de 5 eixos principais (inserção dos estudantes nas atividades práticas; o vínculo da telemedicina com a educação médica à distância; os impactos e adaptações do curso de medicina; as estratégias pedagógicas inseridas ou modificadas durante a pandemia, e os projetos acadêmicos iniciados neste contexto) com o objetivo de esclarecer a realidade atual das Escolas Médicas Brasileiras.

\section{ENSINO MÉDICO EM TEMPOS DE PANDEMIA: CINCO ASPECTOS RELEVANTES.}

\subsection{INSERÇÃO DOS ESTUDANTES NAS ATIVIDADES PRÁTICAS DE ESTÁGIOS CURRICULARES OBRIGATÓRIOS E INTERNATOS MÉDICOS.}


No Brasil, a inserção dos acadêmicos de medicina nas atividades práticas, durante a pandemia, tem se dado, principalmente, nos internatos médicos, devido à obrigatoriedade do estágio prático que esse período da graduação exige das escolas médicas. Estágios curriculares de algumas universidades públicas e privadas também se estenderam para os alunos que cursam o ciclo clínico, ou seja, as aulas práticas passaram a abranger também os alunos de terceiros e quartos anos.

Com relação ao quinto e sexto ano da graduação médica, correspondentes ao internato (estágio curricular obrigatório), as escolas médicas do Brasil, em sua quase totalidade, retomaram os ensinos práticos desses alunos, buscando a manutenção do ensino prático obrigatório exigido pelas Diretrizes Curriculares Nacionais. Esses alunos, então inseridos na prática, entraram como um dos grupos de prioridade com relação à vacinação, quando esta é disponível e possível.

Além do estágio obrigatório propriamente dito, algumas universidades adotaram programas de voluntariado. Destaca-se a Faculdade de Medicina da Universidade de São Paulo (FMUSP), na qual foi facultado aos alunos a possibilidade de auxiliar nas atividades do complexo hospitalar da FMUSP, enquanto não acontecesse a retomada das atividades práticas regulares. A partir de julho de 2020, o quarto ano, também chamado de pré-internato, retomou suas atividades práticas, -em grupos menores e com as devidas precauções- visto que, nesse período, o aluno precisa ser preparado para a atuação eminentemente prática que o período de internato exige, resultado que não é obtido apenas com aulas de ensino à distância (EAD).

Contudo, é importante destacar que essas iniciativas são tomadas a partir de decisões das coordenações e diretorias de cada unidade acadêmica, faculdade e universidade em questão e em conjunto; haja vista que as Instituições de Ensino Superior têm autonomia didático-científica para elaborar o seu programa nos termos do art.207 da Constituição da República Federativa do Brasil de 1988.

\subsubsection{A necessidade da prática}

De uma forma geral, incluindo alunos do ciclo clínico e do internato, a educação médica vivenciada na prática, ao possibilitar a aplicação dos conhecimentos obtidos através do ensino teórico, é de fundamental importância para a aquisição das habilidades necessárias ao cuidado com o paciente, o que será exigido dos futuros profissionais quando formados (BATISTA \& BATISTA, 2008; BULCÃO, 2003). A possibilidade de aulas práticas auxilia de 
forma essencial a formação dos acadêmicos de medicina, pois neste momento os estudantes lidam com pacientes sob supervisão e acompanhamento de profissionais médicos, tendo a oportunidade de aprender sobre as tomadas de decisões e as dinâmicas dos serviços de saúde, por exemplo.

O ensino médico deve ser pautado em competências preciosas, tais como a responsabilidade pessoal e o profissionalismo. Elas são fundamentais para o cuidado adequado com a vida humana, o que que se inicia nas universidades e escolas médicas e se potencializa com o aprendizado prático. Tinto (1998) e Polydoro (2000), corroboram com a ideia ao entenderem a inserção do estudante na prática como parte fundamental da formação, na qual há possibilidades para o desenvolvimento de diferentes aprendizagens e habilidades. Segundo os autores, essa vivência possibilita socialização e estruturação da identidade, formando-se como profissional a partir das interações com colegas, professores, equipe de saúde e comunidade de uma forma geral.

\subsubsection{O risco envolvido nas aulas práticas na pandemia}

Embora o aprendizado prático seja um elemento essencial para formação médica, há um inegável risco de contaminação dos estudantes, médicos e pacientes pelo Sars-Cov 2, em vista da exposição destes ao vírus em hospitais, unidades básicas e centros de saúde. Desta forma, seria necessário o respeito a um criterioso Plano de Biossegurança, atento a todos os detalhes minuciosos e que minimizasse, da melhor forma possível, os riscos da contaminação, tornando as práticas mais seguras.

\section{2. A EXISTÊNCIA DA EDUCAÇÃO MÉDICA A DISTÂNCIA E O SEUVÍNCULO COM A PRÁTICA DE TELEMEDICINA}

De acordo com o Conselho Federal de Medicina (CFM), a telemedicina é definida como "o exercício da medicina mediado por tecnologias para fins de assistência, educação, pesquisa, prevenção de doenças e lesões e promoção de saúde." (CFM, Resolução no 2.227/2018). Para relacioná-la ao Ensino médico on-line, precisamos, entretanto, saber da sua origem. A história da Telemedicina no Brasil permeia o ano de 1994 com o surgimento do "Eletrocardiograma à distância" pela empresa Telecardio. No ano seguinte, a empresa InCor lança o ECG-Fax, onde médicos da própria empresa fariam a leitura e interpretação de Eletrocardiogramas que receberam por meio de Fax. Assim, a Medicina on-line, está na 
realidade do povo Brasileiro há quase três décadas. (CORRÊA, et al., 2020).

Mesmo possuindo uma história considerável no Brasil, somente durante a pandemia de SARS-CoV-2, o Senado Federal aprovou a Lei No 13.989, de abril de 2020, posteriormente sancionada pelo Presidente em exercício. Esta lei regulamenta, no decorrer deste episódio mundial, "o exercício da medicina mediado por tecnologias para fins de assistência, pesquisa, prevenção de doenças e lesões e promoção de saúde" (BRASIL, LEI N ${ }^{0}$ 13.989, 2020, p. 1).. Após o período de crise instaurado em decorrência da COVID-19, o Conselho Federal de Medicina será incumbido de regulamentar a prática.

Atualmente, a Medicina caminha a passos largos para atendimentos remotos, quando (e somente quando) o exame físico não é extremamente necessário, uma vez que "a teleconsulta pode inviabilizar o exame físico, pois o médico estará longe do paciente”. (LOPES, et al, 2020, p. 461). A própria Lei $\mathrm{N}^{\circ} 13.989$ declara que há limitações inerentes ao uso da telemedicina, relacionados "à impossibilidade de realização de exame físico durante a consulta" (BRASIL, 2020). Este é, portanto, o ponto crucial de divergência da Educação Médica à distância e a prática da Telemedicina. Sendo assim, a prática da telemedicina é regulamentada e pode ser utilizada em pacientes com acompanhamento, sem lesões e para os quais o exame físico, (através de ausculta e palpação, por exemplo), não seja crucial.

A telemedicina, embora possa ser usada proveitosamente na educação médica, possui uma série de limitações, e o aluno que possui uma carga horária exclusivamente remota não consegue suprir a necessidade de aulas práticas por estar impossibilitado de frequentar Unidades Básicas de Saúde ou Hospitais Universitários que trariam essa experiência.

Para um médico ter sua experiência acadêmica de sucesso e de excelência, ele precisa, no mínimo, sair da graduação realizando uma consulta digna. Ou seja, ele precisa seguir os atos da consulta que, de acordo com o CFM, por intermédio da Resolução CFM 1.958/2010 (p. 1), consiste em realizar

\footnotetext{
“(...) a anamnese, o exame físico e a elaboração de hipóteses ou conclusões diagnósticas, solicitação de exames complementares, quando necessários, e prescrição terapêutica como ato médico completo e que pode ser concluído ou não em um único momento" (RESOLUÇÃO CFM N ${ }^{\circ} 1.958 / 2010$, p. 01)
}

A educação médica deve possibilitar aos estudantes a oportunidade de realizar na prática, sob supervisão, os procedimentos acima, necessários para o aprendizado das competências profissionais para realização de uma consulta médica digna. Nesse sentido, 
argumentamos que o ensino à distância, sozinho, ainda que se utilize das ferramentas da Telemedicina, não é capaz de formar um médico que possa desenvolver uma consulta com o mínimo de qualidade proposto.

\subsection{IMPACTOS E ADAPTAÇÕES DOS CURSOS DE MEDICINA}

O ensino médico durante a pandemia da COVID-19 mudou drasticamente. Em apenas alguns meses, discentes e docentes tiveram que adaptar suas rotinas para um sistema de educação a distância, com diminuição ou abolição das práticas presenciais em todos os ciclos dos cursos de medicina. Como cada ciclo possui sua singularidade e seu objetivo específico na formação médica, abordaremos as repercussões das mudanças em cada um individualmente, para melhor esclarecimento e visão.

É importante salientar que as escolas médicas do país utilizam-se de diferentes métodos de ensino, a saber: a metodologia tradicional, as metodologias ativas ou, até mesmo, uma forma mista que integra elementos das duas metodologias. As metodologias tradicionais baseiam-se em um ensino centralizado na figura do professor e em sua experiênciaacadêmica e clínica que procura uma excelência técnica e científica. As aulas são ministradas para grandes grupos, geralmente em forma de palestras, com grande quantidade de conteúdo passado ao aluno pelo professor (ABREU, 2009). Assim, o estudante tem uma atuação passivaem seu aprendizado. Essa metodologia ainda está presente em muitas instituições de ensino nopaís, porém vem se modificando ao longo do tempo para se adaptar a uma metodologia mais ativa, como é o caso de instituições mais antigas, são exemplos a Universidade Federal de Goiás (UFG) e a Universidade de São Paulo (USP).

A metodologia ativa, por sua vez, coloca o estudante como o centro da aprendizagem e o professor como facilitador do processo de ensino. Essa forma de aprendizagem pode apresentar-se de várias maneiras, as mais presentes nas escolas médicas brasileiras são a Aprendizagem Baseada em Problemas (PBL) e a Aprendizagem Baseada em Equipe (TBL) (FARIAS, et al., 2015). Em ambas o aprendizado é construído com atividades como discussão de casos clínicos, resolução de problemas e atividades em equipe que integra diferentes matérias em que há uma discussão ativa entre professor e aluno para a construção do conteúdo. Essa metodologia está presente em faculdades como a Universidade Estadual de Londrina (UEL), uma das primeiras a implantar esse método de ensino, e em várias das novíssimas faculdades de medicina, que surgiram depois de 2014. 


\subsubsection{Ciclo Básico}

O ciclo básico inclui os dois primeiros anos da faculdade de medicina e engloba matérias básicas relacionadas às ciências biológicas, à saúde e às ciências sociais aplicadas. Nesse âmbito, as aulas práticas presentes em matérias importantes como anatomia, histologia, fisiologia e outras, foram as mais prejudicadas pela suspensão das aulas. Em faculdades em que a metodologia utilizada antes da pandemia era a metodologia tradicional, houve uma adaptação do ensino para o uso de metodologias ativas. Deste modo, as práticas foram substituídas por métodos que podem ser adaptados para o sistema remoto como discussões de casos clínicos (OLIVEIRA, 2020). Essa estratégia, entretanto, não possibilita a confluência entre teoria e prática esperada para esses períodos acadêmicos.

\subsubsection{Ciclo Clínico}

O ciclo clínico consiste no $3^{\circ}$ e $4^{\circ}$ anos do curso, sendo o momento em que o aluno inicia maior contato com as especialidades médicas. Esse período tem como finalidade formar habilidades profissionais essenciais através de discussões teóricas sobre temas associados a práticas em ambulatório. O ciclo clínico foi intensamente prejudicado pelo ensino remoto, devido à suspensão temporária das aulas das escolas médicas no período inicial da pandemia. Tal fato prejudicou a consolidação do ensino teórico, pois os alunos não puderam treinar o que aprenderam nas aulas.

Pela ampliação do período pandêmico, algumas faculdades optaram por retornar às atividades presenciais quando esse serviço era permitido pelas legislações municipais. Essas atividades eram preconizadas a serem feitas em grupos menores e com todas as medidas de proteção necessárias. O retorno era algo a ser optado pelo aluno, além de que as faculdades sugeriam que os discentes que faziam parte do grupo de risco se mantivessem em casa, como feito pela faculdade de Ciências Médicas (MG) em 17 de junho de 2020 (OLIVEIRA, 2020).

Os alunos que optaram pelo ensino híbrido se depararam com quantidade insuficiente de equipamentos de proteção individual (EPIs), com diversos horários de ambulatórios cancelados (devido à baixa adesão dos pacientes), além de testemunharem o despreparo em relação a biossegurança nos ambientes de assistência primária e hospitalar. Essas situações foram observadas por estudantes de medicina da Uniderp em Campo Grande (MS) (ZURUTUZA, 2021). 
Ao final de 2021 e início de 2021, com a melhora da situação epidemiológica em certos municípios, algumas faculdades decidiram disponibilizar a possibilidade do retorno totalmente presencial aos alunos, como Unifenas em Minas Gerais. Novamente, foram observadas falhas das instituições, como aglomerações em salas de aula e desrespeito às condições sanitárias vigentes, o que foi relatado por acadêmicos da Universidade de Nova Iguaçu (RJ) (HENDERSON, 2021).

\subsubsection{Internato}

Já o internato, consiste nos estágios curriculares obrigatórios realizados nos últimos dois anos da graduação, o que corresponde a cerca de 35\% da carga horária dos cursos, em média. Inicialmente, os alunos de medicina nessa fase do ensino se viram frente a um impasse: deveriam escolher entre a antecipação da formatura (para contribuir na linha de frente ao combate a COVID-19), ou aguardarem fim do período de suspensão das práticas médicas para completarem o curso com $100 \%$ da carga horária.

A grande maioria dos alunos que haviam feito $75 \%$ da carga do internato, e que optaram pela antecipação, passou a ocupar parte das vagas na atenção primária, logo após a colação feita no ambiente virtual. Aqueles que se mantiveram na instituição, ou que ainda não se enquadraram na carga horária para antecipar a colação de grau, tiveram o retorno das suas práticas quando as medidas sanitárias permitisseme/ou quando as instituições de ensino superior asseguravam a segurança dos seus alunos.

Contudo, esses alunos enfrentam dificuldades, assim como os alunos do ciclo clínico. Os internos entraram, na grande maioria das cidades, no grupo prioritário de vacinação, devido aoseu contato direto com pacientes infectados pelo Novo Coronavírus. Desta forma, aqueles quetiveram sua vacinação assegurada foram resguardados quanto ao risco de uma infecção mais severa. Entretanto, não há certeza sobre um potencial vacinação dos alunos dos demais períodos $\left(1^{\circ}\right.$ ao $\left.8^{\circ}\right)$, mesmo com a possibilidade do início das aulas práticas presenciais. Essa incerteza évista no Distrito Federal, onde apenas os internos que atuam no Hospital Universitário de Brasília foram imunizados até meados de abril de 2021 (DUTRA, 2021).

É importante destacar que a grande maioria dos alunos, independente do período da graduação em que se encontravam, enfrentaram dificuldades em se adaptar às novas formas de ensino adotadas pelas escolas médicas. Os estudantes tiveram que conciliar as limitações do ensino remoto, ou das aulas práticas realizadas fora das condições ideais, com o momento complexo da sociedade, em que suas inseguranças, o medo do contágio e a própria pressão 
exercida sobre aqueles que escolhem a carreira médica dividiam espaço com adaptações na maneira de estudar. Segundo pesquisa publicada no Jornal Brasileiro de Psiquiatria, 80,2\% dos estudantes de medicina de diversas regiões do país relataram perda da concentração durante as aulas online e/ou dificuldade em utilizar as plataformas digitais (TEIXEIRA et al., 2021).

\subsection{ESTRATÉGIAS PEDAGÓGICAS INSERIDAS/MODIFICADAS NA EDUCAÇÃO MÉDICA DURANTE A PANDEMIA DA COVID-19}

Espera-se que os cursos de Medicina estejam comprometidos com a formação de um médico com capacidade de responder às necessidades sociais da população em todos os níveis da atenção à saúde, seja no nível primário (composto pelas Unidades básicas de saúde), seja na atenção secundária (composta pelos serviços especializados encontrados em ambulatórios e hospitais) ou ainda, no nível terciário (composto pelo atendimento de alta complexidade em hospitais de grande porte). Assim, a educação médica deve incluir oportunidade de práticas acadêmicas em unidades da rede de atenção primária, além dos já tradicionais estágios realizados nos hospitais universitários. No entanto, as tensões oriundas da pandemia da COVID-19 afetaram os acadêmicos do curso de medicina, devido à suspensão das práticas. Neste aspecto, as atividades de ensino à distância têm ganhado espaço progressivo na educação superior, sobretudo nos cursos de medicina, buscando facilitar a comunicação do estudante com o docente com o intuito de atenuar os danos do aprendizado presencial devido ao isolamento social.

Com isso, em pouco tempo, as escolas médicas viram-se na necessidade de mudar sua modalidade de ensino para à distância com o intuito de se adaptar à nova realidade e o desafio em manter as atividades educacionais sem comprometer a qualidade de ensino. No Brasil, várias instituições escolares públicas e privadas, atendendo a Portaria nº 343/20209 e a Medida Provisória No 934/202010, substituíram as aulas presenciais por aulas em meios digitais. As instituições de ensino superior privadas buscaram implementar o ensino remoto com aulas transmitidas em tempo instantâneo por sistemas de webconferências. Com isso, essa estratégia de ensino permite que professores e alunos tenham condições de realizar interações e organizar seus tempos de aprendizagem da forma mais próxima à educação presencial.

Logo, é perceptível perceber que os cursos de medicina no Brasil a partir de março de 2020, buscaram estratégias de adaptação no período pandêmico por meio do usode plataforma digitais, diálogo e comunicação entre gestores, professores e alunos com o 
intuito de buscar melhorias para manter a qualidade do ensino-aprendizado e não prejudicar a carga horária do curso de graduação para que o impacto no ensino devido a pandemia seja atenuado.

\subsection{PROJETOS ACADÊMICOS INICIADOS NO CONTEXTO DA PANDEMIA}

As modificações impostas ao processo educacional da formação médica pela pandemia da COVID-19 exigiram um remodelamento emergencial, demandando atenção e diálogo ágil entre educadores, gestores, estudantes e sociedade (OLIVEIRA, et al., 2020).Neste período de adaptações, muitos alunos assumiram o protagonismo e foram responsáveis ou, pelo menos, corresponsáveis pela criação de diversos projetos, que impactaram positivamente tanto o processo de aprendizagem dentro do curso, como a sociedade de modo geral. Neste tópico, mostraremos exemplos desses projetos acadêmicos e iniciativas desenvolvidos por estudantes, abordando sobre seus desdobramentos e importância.

O projeto denominado "Iniciativas de atenção e de educação em saúdepara enfrentamento do novo coronavírus (SARS-CoV-2) no município de Divinópolis, MinasGerais (Projeto PrOVIDa)" é um exemplo de uma iniciativa que surgiu em meio à pandemiae no contexto dessas adaptações do ensino médico no Brasil, ele se mostrou benéfico aos alunos, profissionais de saúde e comunidade envolvidos. O projeto, descrito como de extensão universitária, envolveu alunos do curso de Medicina da Universidade Federal de São João Del Rei - Campus Centro

Oeste Dona Lindu (UFSJ/CCO) sob supervisão de professores médicos do curso, e se propôs, por meio da realização de treinamentos com simulação realística, a trocar informações com profissionais de saúde e a população por meio da integração ensino-serviçocomunidade, a fim de alinhar conhecimentos para melhor capacitar as equipes da rede de Atenção Primária à Saúde (APS) no enfrentamento da epidemia do SARS-CoV-2 no município (OLIVEIRA DOS SANTOS, et al., 2020).

Outra modalidade de projetos organizados por alunos surgida no último ano,em decorrência das necessidades impostas pelo distanciamento social e suspensão das aulas presenciais, é a dos cursos e eventos online. O termo "inundação" de cursos, congressos e eventos online foi utilizado (MARSILLI, et al., 2021), e, apesar de levantar questionamentos a respeito da sobrecarga de horas e informações, visto que qualquer estudante de medicina reconhece a importância de participar desses eventos para a construção de seu currículo 
(MARSILLI, et al., 2021), essa modalidade de estudo e ensino abriu portas para uma significativa possibilidade de compartilhar o conhecimento de maneira acessível e prática.

As perdas e consequências negativas ao processo educacional da formação médica brasileira nesse período de pandemia da COVID-19 são inúmeras e evidentes (CHINELATTO et al., 2020). A China, entretanto, provou que projetos e adaptações desenvolvidas nesse contexto podem ser continuados por contribuírem positivamente com o ensino médico (AHMED et al., 2020). Nesse sentido, haja vista seus benefícios para a comunidade, serviços de saúde e formação profissional, espera-se que os projetos de extensão universitária, assim comoos eventos e cursos online, por viabilizarem fácil acesso e troca de conhecimentos, sejam valorizados como importantes contribuições dos alunos e continuem sendo implementados nos anos subsequentes.

\section{CONSIDERAÇÕES FINAIS}

A pandemia da COVID-19 se constitui uma das mais impactantes questões de saúde pública na realidade brasileira e de todo o mundo moderno, em decorrência dos seus impactos sociais. Dentre todas as transformações resultantes do cenário pandêmico, a interferência abrupta no processo educacional da formação médica é inegável, e exigiu um remodelamento emergencial, demandando atenção e diálogo ágil entre educadores, gestores, estudantes e sociedade (OLIVEIRA, et al., 2020).

O padrão do ensino de modo geral está em uma constante evolução (e ainda foi influenciada pelo atual contexto):

Híbrido significa misturado, mesclado, blended. A educação sempre foi misturada, híbrida, sempre combinou vários espaços, tempos, atividades, metodologias, públicos. Esse processo, agora, com a mobilidade e a conectividade, é muito mais perceptível, amplo e profundo: é um ecossistema mais aberto e criativo. Podemos ensinar e aprender de inúmeras formas, em todos os momentos, em múltiplos espaços. Híbrido é um conceito rico, apropriado e complicado. Tudo pode ser misturado, combinado, e podemos, com os mesmos ingredientes, preparar diversos “pratos”, com sabores muito diferentes. (MORAN. 2015, p. 22)

Na relação direta com a medicina, argumentamos que as práticas presenciais ainda são estritamente necessárias. Podemos tomar como exemplo o estudo da anatomia. Segundo 
Salbego et al., (2014), ele é "fundamental para possibilitar o reconhecimento dos órgãos do corpo humano, assim como a morfologia, localização, função e organização desses órgãos em sistemas" (SALBEGO et al., 2014 p. 7). Considerando Salbego e Moran, o ensino híbrido é a saída para a Medicina (e outros cursos da saúde que demandam a mesma necessidade) em tempos excepcionais.

A educação médica no Brasil visualizada a partir do ano de 2020 tem, portanto, potencial de avançar para o Ensino híbrido, respeitando a segurança de todos, desde discentes, docentes, funcionários terceirizados e demais, e que as Escolas médicas respeitem o momento prático presencial e incorporem as novas tecnologias educacionais como elementos auxiliares para um processo de ensino-aprendizagem efetivo. 


\section{REFERÊNCIAS BIBLIOGRÁFICAS:}

ABREU, José Ricardo Pinto. Contexto Atual do Ensino Médico: Metodologias Tradicionais e Ativas - Necessidades Pedagógicas dos Professores e da Estrutura das Escolas. Tese (Mestrado em Medicina) - Faculdade de Medicina, Universidade Federal do RioGrande do Sul, Porto Alegre, p. 175, 2009

AHMED, Alsoufi et. al. Impact of the COVID-19 pandemic on medical education: Medical students' knowledge, attitudes, and practices regarding electronic learning. Plosone. 25 Nov. 2020. Disponível em: <https://doi.org/10.1371/journal.pone.0242905> . Acesso em: 20/04/2021.

BERNARDES, Júlio. Na Pandemia, curso de Medicina da USP adota aulas virtuais e voluntariado. Jornal da USP, São Paulo - SP, 24 jul. 2020. Disponívelem: <https://jornal.usp.br/?p=340385>. Acesso em: 20/04/2021.

BRASIL. Lei $n^{\circ} 13.989$, de 16 de Abril de 2020. Dispõe sobre o uso da telemedicina durante a crise causada pelo coronavírus (SARS-CoV-2). Disponível em: <https://www.in.gov.br/en/web/dou/-/lei-n-13.989-de-15-de-abril-de-2020- 252726328>. Acesso em: 20/04/2021.

BRASIL. Ministério da Educação. Portaria MEC n ${ }^{\circ} 1.038$, de 7 de Dezembro de2020. Disponível em <https://www.in.gov.br/en/web/dou/-/portaria-mec-n-1.038-de-7-de- dezembro de-2020-292694534>. Acesso em: 20/04/2021

BRASIL. Portaria № 323, de 15 de Março de 2020. Dispõe sobre a substituição dasaulas presenciais por aulas em meios digitais enquanto durar a situação de pandemia doNovo Coronavírus - COVID-19. Disponível em: <https://www.in.gov.br/en/web/dou//portaria-n 343-de-17-de-marco-de-2020-248564376>. Acesso em: 20/04/2021.

CHINELATTO, Lucas Albuquerque et al., What You Gain and What You Lose in COVID 19: Perception of Medical Students on their Education. Clinics. Clinics, vol. 75. Jul. 2020. Disponível em: https://doi.org/10.6061/clinics/2020/e2133

CHRISTENSEN, Clayton Magleby; HORN, Michael; STAKER, Heather. Is K-12 Blended Learning Disruptive? An introduction to the theory of hybrids. Clayton Christensen Institute, maio 2013.

CONSELHO FEDERAL DE MEDICINA (CFM-Brasil). Resolução CFM 2.227/2018: Define e disciplina a telemedicina como forma de prestação de serviços médicos mediados por tecnologia.

Disponível em: 
<https://portal.cfm.org.br/images/PDF/resolucao222718.pdf>. Acesso em: 20/04/2021.

COOK, David; LEVINSON, Anthony; GARSIDES, Sarah; DENISE, Dupras; ERWIN, Patricia; MONTORI, Victor. Intructional design variations in internet-based learning for health professions education: A systematic review and meta-analysis. Academic Medicine. 85:909-922, May. 2010.

CORRÊA, J. C. B. et al., Telemedicina no Brasil: Desafios ético-jurídicos em tempos de pandemia da Covid-19. Humanidade e Tecnologia (FINOM) V. 25, N. 1. 2020.

DUTRA, Francisco. COVID-19: na linha de frente, 1.570 alunos da área da Saúde pedem vacina. 2021. Disponível em: https://www.metropoles.com/distrito-federal/covid-na-linha-defrente-1-570-alunos-da-area-da-saude-pedem-vacina. Acesso em: 14 maio 2021.

FARIAS, Pablo Antônio Maia; MARTIN, Ana Luiza de Aguiar Rocha; CRISTO, Cinthia Sampaio. Aprendizagem Ativa na Educação em Saúde: Percurso Histórico e Aplicações. Revista Brasileira de Educação Médica, v. 39, n. 1, p. 143-158, dez./2015. Disponível em: <http://dx.doi.org/10.1590/1981-52712015v39n1e00602014>. Acesso em: 29/04/2021

FERREL, Meganne N; RYAN, John J. The Impact of COVID-19 on Medical Education. Cureus, p. 1-2, 31 mar. 2020.

FREIRE, P. Pedagogia da Autonomia. Rio de Janeiro: Paz e Terra, 1997. JUNIOR, Luiz Severo Bem et. al. Ensino remoto e metodologias ativas na formação médica: desafios na pandemia COVID-19. Jornal Memorial da Medicina, vol. 2 (2), p.44-47. 2020. LAU et. al. Implantação de Estratégias de Ensino à Distância durante o Internato: Desafios e Perspectivas. Revista Brasileira de Educação Médica. Vol.41 Nº.2 Apr./Jun. 2017. Disponível em: $\quad<$ https://www.scielo.br/scielo.php?script=sci_arttext\&pid=S010055022017000200269\#B4>. Acesso em: 24/04/2021.

HENDERSON, Alexandre (ed.). Alunos da Unig reclamam de aglomeração em aulas presenciais e desrespeito a protocolos sanitários. 2021. Disponível em: https://g1.globo.com/rj/rio-de-janeiro/noticia/2021/04/19/alunos-da-unig-reclamam-deaglomeracao-em-as-aulas-presenciais-e-desrespeito-a-protocolos-sanitarios.ghtml. Acesso em: 29 abr. 2021.

MARSILLI, L. R. B.; SMECELLATO, F. B.; SILVA JÚNIOR, O. de C. e. Ensino médico na pandemia de COVID-19: ponto de vista de acadêmicos de medicina. Medicina (Ribeirão Preto), [S. 1.], v. 53, n. 4, p. 490-494, 2020. DOI: 10.11606/issn.2176-7262.v53i4p490494. Disponível em: https://www.revistas.usp.br/rmrp/article/view/174253. Acesso em: 26 abr. 
2021.

MORAN, José Manuel. Educação Híbrida: Um conceito chave para a educação, hoje. In: Ensino híbrido: personalização e tecnologia na educação [recurso eletrônico] / Organizadores, Lilian Bacich, Adolfo Tanzi Neto, Fernando de Mello Trevisani. - Porto Alegre: Penso, 2015. e-PUB.

OLIVEIRA DOS SANTOS, A.; CRISTIAN DO AMARAL, P.; FERNANDES DE MOURA PIRES, B.; MACHADO ROCHA, G.; CABRAL SILVA, H. Percepções de estudantesde medicina e profissionais de saúde sobre a capacitação de equipes da atenção primáriaà saúde no enfrentamento da epidemia da COVID-19. Revista Brasileira de Extensão Universitária, v. 11, n. 2, p. 227-236, 3 ago. 2020.

OLIVEIRA, Natália. Faculdade Ciências Médicas decide voltar às atividades, e alunos se revoltam. 2021. Disponível em: https://www.otempo.com.br/cidades/faculdade-cienciasmedicas-decide-voltar-as-atividades-e-alunos-se-revoltam-1.2348621. Acesso em: 29 abr. 2021.

OLIVEIRA, Sandro Schreiber; POSTAL, Eduardo Arquimino; AFONSO, Denise Herdy. As Escolas Médicas e os desafios da formação médica diante da epidemia brasileira da Covid19: das (in)certezas acadêmicas ao compromisso social. APS em revista, v.2, n. 1, p, 56-60, abr. $/ 2020$

POLYDORO, S. A. J. 2000. O Trancamento de Matrícula na Trajetória Acadêmica do Universitário: Condições de Saída e de Retorno à Universidade. Tese de Doutorado nãopublicada, Faculdade de Educação, Universidade Estadual de Campinas, Campinas, São Paulo. Recomendações sobre atividades presenciais nas escolas médicas. Associação brasileira de educação médica, Brasília - DF, 15 fev. 2021. Disponível em: <https://website.abemeducmed.org.br/wp-content/uploads/2021/02/Abem-Recomendacoes-sobre-atividades presenciais.pdf>. Acesso em: 20/04/2021.

SALBEGON, Cléton; OLIVEIRA, Elaine Maria Dias de; SILVA, Márcia de Almeirda Rosso; BAGUNÇA, Paula Renata. Percepções Acadêmicas sobre o Ensino e a Aprendizagem em Anatomia Humana. Revista Brasileira de Educação Médica. vol.39, n.1, pp.23-31. Mar. 2015. SAÚDE MENTAL DOS ESTUDANTES DE MEDICINA DO BRASIL DURANTE A PANDEMIA DA CORONAVIRUS DISEASE 2019. Rio de Janeiro, 31 mar. 2021. Disponível em: https://www.scielo.br/scielo.php?script=sci_arttext\&pid=S004720852021000100021. Acesso em: 14 maio 2021. 
TINTO, V. 1998. Stages of Student Departure. Reflections on the Longitudinal Character of Student Leaving. Journal of Higher Education, 59 (4), pp. 438-455.

UNIFENAS (Brasil). ORIENTAÇÕES PARA RETOMADA DAS ATIVIDADES ACADÊMICAS PRESENCIAIS AOS ALUNOS DO CÂMPUS DE ALFENAS, DA UNIFENAS EM CONFORMIDADE COM A PORTARIA MEC No 1.038, DE 7 DE DEZEMBRO DE 2020. 2020. Disponível em: unifenas.br/retomadapraticas.asp. Acesso em: 29 abr. 2021.

ZURUTUZA, Anahi (ed.). Acadêmicos de Medicina reclamam de atender pacientes com covid. 2021. Disponível em: https://www.campograndenews.com.br/direto-dasruas/academicos-de-medicina-reclamam-de-atender-pacientes-com-covid. Acesso em: 29 abr. 2021. 